\title{
Accuracy of MRCP compared with ERCP in the diagnosis of bile duct disorders
}

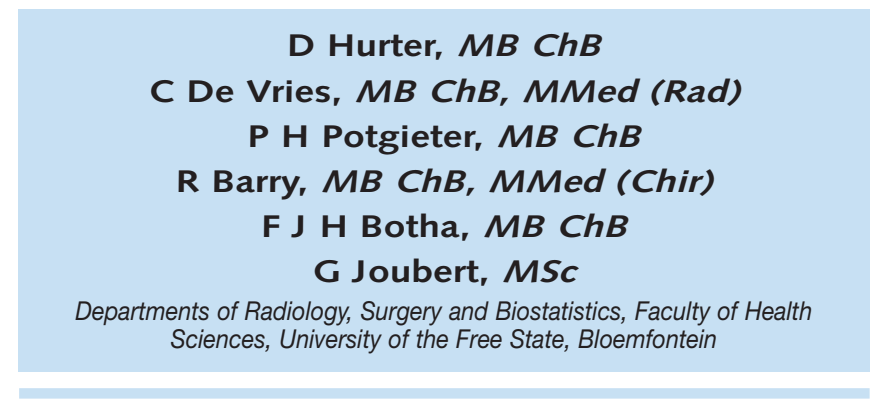

\begin{abstract}
Aim. To determine the accuracy of magnetic resonance cholangiopancreatography (MRCP) compared with the gold standard endoscopic retrograde cholangiopancreatography (ERCP) in the diagnosis of bile duct disorders at Universitas Hospital, University of the Free State (UFS), Bloemfontein.

Patients and methods. Fifty-two patients with suspected pancreatobiliary pathology were included in this prospective observational study. MRCP was performed in the 24-hour period prior to ERCP.

Results. MRCP had sensitivity, specificity, positive and negative predictive values of $87 \%, 80 \%, 83.3 \%$ and $84.2 \%$ respectively for choledocholithiasis, which correlates well with results obtained in other parts of the world.

Conclusion. At UFS, MRCP has high diagnostic accuracy for bile duct calculi. Owing to a small study population, results for other biliary pathology were inconclusive.
\end{abstract}

\section{Introduction}

Accurate methods for detecting bile duct and pancreatic duct abnormalities in patients with obstructive jaundice are important to both surgeons and endoscopists. Biliary obstruction may be the result of choledocholithiasis, tumours or trauma, among other causes. The most common cause is choledocholithiasis.

ERCP is still the gold standard for exploration of the biliopancreatic region. However, it requires direct cannulation of the common bile or pancreatic duct, sedation, the use of ionising radiation and a team of trained and experienced personnel. In addition, ERCP is associated with significant complication rates of $1-7 \%$, such as haemorrhage, sepsis, pancreatitis and bile leak, as well as a recognised mortality of up to $1 \%{ }^{1}$

MRCP is a non-invasive and safe alternative to diagnostic ERCP for imaging the biliary tree and investigating biliary obstruction. MRCP refers to selective fluid-sensitive magnetic resonance imaging (MRI) of the pancreatic and biliary ducts. It was developed in 1991 and techniques have progressively improved since then.

A major detriment or disadvantage of MRCP is that it is not a therapeutic procedure, whereas ERCP is used for diagnosis and treatment.
However, if no therapeutic intervention is found to be necessary, MRCP avoids the potential morbidity and mortality associated with ERCP. MRCP is particularly useful where ERCP is difficult, hazardous or impossible. It is also an important option for patients with failed ERCPs. ERCP and MRCP have different contraindications, allowing them to be used as complementary techniques. ${ }^{2}$

\section{Aim}

The aim of our study was to compare the accuracy of MRCP as a diagnostic tool at our institution with invasive ERCP in the diagnosis of bile duct abnormalities, using specificity, sensitivity, and positive and negative predictive values.

\section{Materials and methods}

During the period May 2003 to November 2004, 52 patients with suspected pancreaticobiliary pathology and a clear indication for ERCP were included in this prospective study. Patients had to be at least 18 years old, and informed written consent was obtained prior to both procedures.

Our study was run at the MRI and the ERCP units of Universitas Hospital, UFS, Bloemfontein. All the patients had both examinations performed within 24 hours of each other. Patients with absolute contraindications to the MRCP technique (e.g. cardiac pacemaker, claustrophobia, large patient size, degenerative or ankylotic conditions or senile dementia) were excluded from the study because of the impossibility of their co-operation, as were patients with severe clinical conditions requiring urgent therapeutic intervention.

\section{MRCP and MRI technique}

MRCP was performed on a $1.5 \mathrm{~T}$ General Electric unit (Signa) using a torso phased-array coil.

1. Three plane gradient-echo localising images were obtained and used to plan MRCP sequence.

2. Axial slices were performed using single-shot fast spin-echo (SSFSE) sequences; parameters: $90 \mathrm{TE}$.

- Field of view $28-38 \mathrm{~cm}$

- Slice thickness: $10 \mathrm{~mm}$

- Spacing: $2 \mathrm{~mm}$

- Frequency: $256 \mathrm{kHz}$

- Phase encoding: Field of view (FOV): $8 \mathrm{~cm}$

- Frequency encoding direction: right to left

3. Radial slice acquisitions with high resolution, thick slab using long TE were performed in the region of the biliary and pancreatic ducts. Eleven reconstructed slices with 10-degree spacing were used.

4. Coronal $3 \mathrm{~mm}$ FOV - spacing $3 \mathrm{~mm}$.

All the sequences were acquired during a single breath-hold after a 4 - 6-hour period of fasting to promote gallbladder filling. The entire examination was usually completed within 20 minutes. 


\section{ERCP technique}

Fluoroscopy: A Philips Diagnost 93 was used for screening and taking hard copy plain films.

Duodenoscopy: An Evis Olympus JF type 230 duodenovideoscope was used.

The procedure was performed with patients under conscious sedation or general anaesthesia, depending on the individual evaluation of the patients by the anaesthetist. Patients were positioned in the prone position and ERCP performed by an experienced surgical endoscopist. The endoscopist had no access to information from the prior MRCP

\section{Image analysis}

The diagnostic quality, coverage of the relevant anatomy and results of the MRCP were reviewed by one general radiologist blinded to the ERCP results. He received only clinical information related to the symptoms of patients.

The ERCP was interpreted by an experienced consultant surgeon also blinded to the MRCP results. Results for cholangiography and pancreatography obtained from both techniques were compared. Results were analysed according to the pathology found, e.g. choledocholithiasis, pancreaticobiliary strictures and dilatation.

\section{Statistical analysis}

The sensitivity, specificity, and positive and negative predictive values were used to compare the two imaging techniques. Analysis focused on the comparison of cholangiography and pancreatography as obtained by both techniques. Fisher's $2 \times 2$ exact test was used to compare groups. Statistical significance was set at $p<0.05$, with $95 \%$ confidence intervals.

\section{Results}

Patients' ages ranged from 21 to 86 years, with a mean of 60.5 years. Thirty-two patients (61.5\%) were female and 20 patients (38.5\%) were male. Patients were evaluated for clinical and biochemical jaundice: 32 patients (65.3\%) and 41 patients (83.7\%) were clinically and biochemically jaundiced, respectively. In 3 patients, these results were not obtainable (Table I).

\section{Table I. Patient demographics}

\begin{tabular}{ll}
\hline Total number of patients $(N)$ & 52 \\
Female & $32(61.5 \%)$ \\
Male & $20(38.5 \%)$ \\
Age & $21-86$ years (mean \\
& $60.5)$ \\
Jaundice & \\
$\quad$ Clinically & $32(65.3 \%)$ \\
Biochemically & $41(83.7 \%)$ \\
Unknown & 3
\end{tabular}

\section{ERCP}

Choledocholithiasis was found in 26 patients; in these, the bile ducts were involved in 23 patients and the ampulla of Vater in 3 patients. Strictures were diagnosed in 15 patients; 12 were seen in the bile ducts,
2 were found in the pancreatic duct and 1 was thought to be due to a tumour in the main papilla. Dilated bile ducts were found in 34 patients in conjunction with either stones or strictures. The ERCP was normal in 3 patients.

ERCP failed in 5 patients due to papillary oedema, impacted stone just above the ampulla, duodenal tumour, pharyngeal tumour and patient non-compliance.

\section{MRCP}

Choledocholithiasis was seen in 31 patients, affecting the ampulla of Vater in 7 patients and the bile ducts in 24 patients. Strictures were diagnosed in 9 patients; 3 owing to suspected tumours in the main papilla, 5 were seen in the bile ducts and 1 was found in the pancreatic duct. Dilated bile ducts were found in 20 patients in conjunction with either stones or strictures. The MRCP was normal in 3 patients. MRCP failed in 1 patient due to artifacts caused by surgical clips. Two subjects had ascites, leading to failed MRCP due to lack of visibility of the ducts.

\section{MRCP v. ERCP (Table II)}

MRCP correctly diagnosed 20 of the 23 patients with ERCP-proven bile duct calculi, and 16 of the 20 patients without calculi. There were 3 false-negative and 4 false-positive results for MRCP. Stones in the ampulla of Vater were correctly diagnosed in 1 of the 3 affected patients, and excluded in 35 of the 41 patients. There were 6 false-positive and 2 false-negative results when MRCP was used to diagnose stones in the ampulla.

The sensitivity, specificity, and positive and negative predictive values for bile duct calculi were $87 \%, 80 \%, 83.3 \%$ and $84.2 \%$, respectively.

Strictures were correctly diagnosed by MRCP in 4 of the 12 affected patients, and excluded in 28 of the 29 unaffected patients. There were 1 false-positive and 8 false-negative results for MRCP. The sensitivity, specificity, positive and negative predictive values were $33.3 \%, 96.6 \%$, $80 \%$ and $77.8 \%$, respectively.

MRCP correctly diagnosed 1 of the 2 patients with bile duct occlusion, and 30 of the 38 patients without occlusion. There were 1 false-negative and 8 false-positive results for MRCP in the diagnosis of occlusion of the bile ducts. In this instance, the sensitivity, specificity, and positive and negative predictive values were $50 \%, 78.9 \%, 11.1 \%$ and $96.8 \%$, respectively.

Bile duct dilatation was found by MRCP in 18 of the 34 affected patients and excluded by MRCP in 7 of the 9 unaffected patients. There were 2 false-positive and 16 false-negative results for MRCP. The sensitivity, specificity, and positive and negative predictive values were $52.9 \%, 77.8 \%, 90 \%$ and $30.4 \%$, respectively.

Ampullary tumours were correctly diagnosed in the only affected patient, but 2 false-positive results were found. MRCP failed to diagnose all patients with bile duct leaks.

The overall sensitivity, specificity, and positive and negative predictive values for bile duct pathology were $60.5 \%, 84.3 \%, 74.1 \%$ and $74.3 \%$, respectively.

The sensitivity and positive predictive values for pancreatic duct filling defects were $100 \%$. Regarding the diagnosis of pancreatic duct strictures, a sensitivity of $100 \%$, specificity of $50 \%$, positive predictive value of $94.1 \%$, and negative predictive value of $100 \%$ were found. 
Table II. Main specificity and sensitivity results

\begin{tabular}{|c|c|c|c|c|c|}
\hline System & Diagnostic feature & Sensitivity (\%) & Specificity (\%) & Positive predictive value (\%) & $\begin{array}{l}\text { Negative predictive } \\
\text { value }(\%)\end{array}$ \\
\hline \multirow{2}{*}{ Main papilla } & Tumour present & 100 & 95.3 & 33.3 & 100 \\
\hline & Stones & 33.3 & 85.4 & 14.3 & 94.6 \\
\hline \multirow[t]{4}{*}{ Bile ducts } & Stones & 87 & 80 & 83.3 & 84.2 \\
\hline & Dilated & 52.9 & 77.8 & 90 & 30.4 \\
\hline & Strictures & 33.3 & 96.6 & 80 & 77.8 \\
\hline & Occlusion & 50 & 78.9 & 11.1 & 96.8 \\
\hline Gallbladder & Stones & 80 & 50 & 80 & 50 \\
\hline \multirow[t]{3}{*}{ Pancreatic duct } & Occlusion & & 90 & & 94.7 \\
\hline & Filling defects & & 100 & & 100 \\
\hline & Strictures & 50 & 100 & 100 & 94.1 \\
\hline
\end{tabular}

\section{The following MRCP and ERCP images demonstrate stones and strictures.}

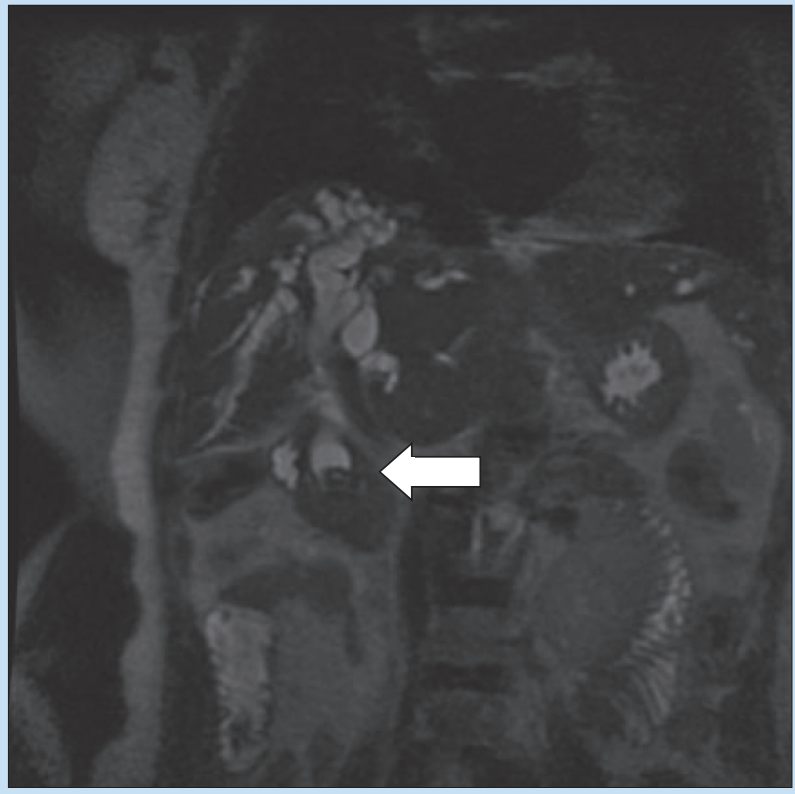

Fig. 1. Coronal single-shot fast spin-echo T2 MRI demonstrating a lowintensity stone (white arrow) impacted in the common bile duct.

In all of the patients with failed MRCP, ERCP was 100\% successful. ERCP failed in 1 patient due to papillary oedema; in this case, MRCP successfully diagnosed choledocholithiasis and secondary bile duct dilatation. In the patient with the stone impacted just above the ampulla and the other patient with the pharyngeal tumour, MRCP was able to show the anatomy of the remaining duct system. MRCP also failed in the patient with the duodenal tumour, due to severe ascites.

In addition, ERCP allowed therapeutic intervention with sphincterotomy, stone extraction and/or stent placement. Where relevant, histology could also be obtained.

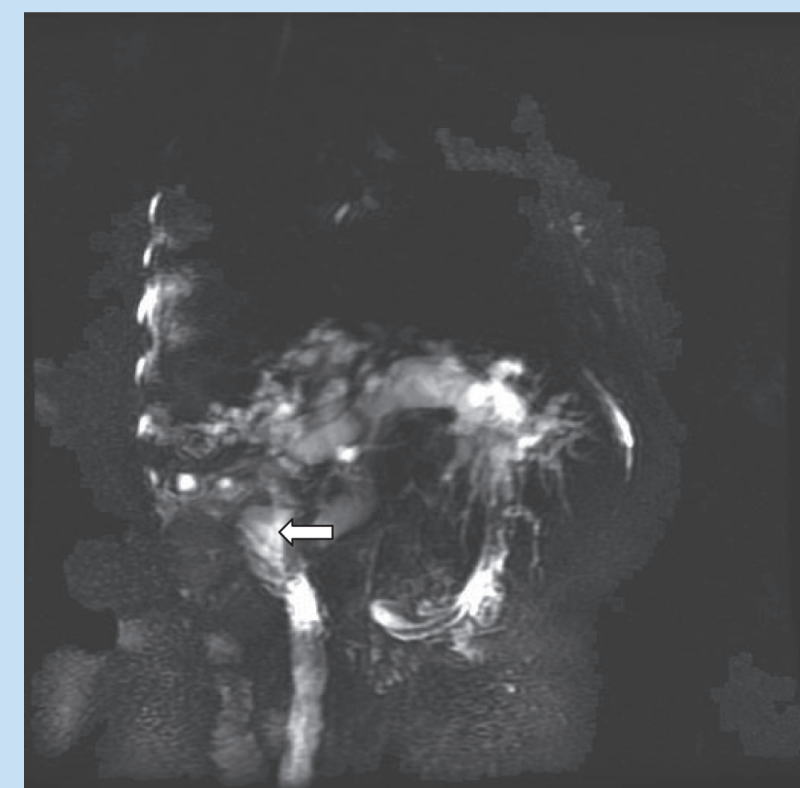

Fig. 2. High-resolution thick slab long TE $1100 \mathrm{~ms}$ (rotated to RAO position) MRCP demonstrating a stone impacted in the common bile duct (white arrow).

\section{Discussion}

The aim of our study was to compare the accuracy of MRCP as a diagnostic tool at our institution with invasive ERCP in the diagnosis of bile duct abnormalities, using specificity, sensitivity, and positive and negative predictive values. If these values were favourable for MRCP, then the latter could be proposed as the examination of choice for diagnostic imaging of bile duct abnormalities, and ERCP could then be reserved for therapeutic intervention alone.

MRCP is a non-invasive and safe alternative to ERCP for imaging the biliary tree and investigating biliary obstruction. Even though 


\section{ORIGINAL ARTICLE}

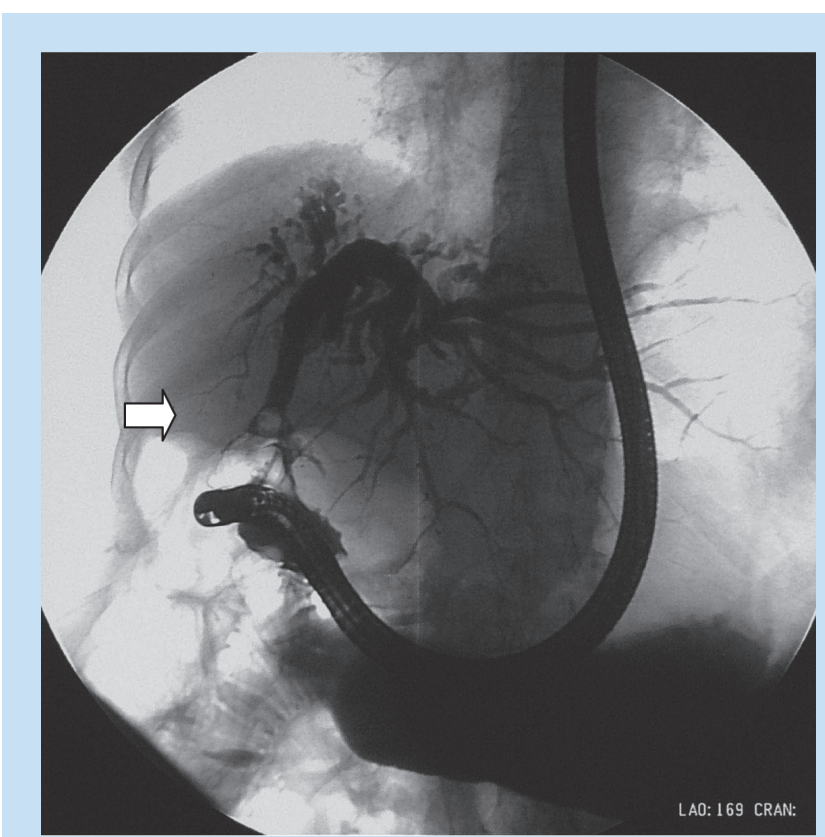

Fig. 3. ERCP image (LAO view) demonstrating a stone impacted in the common bile duct (white arrow) in the same patient.

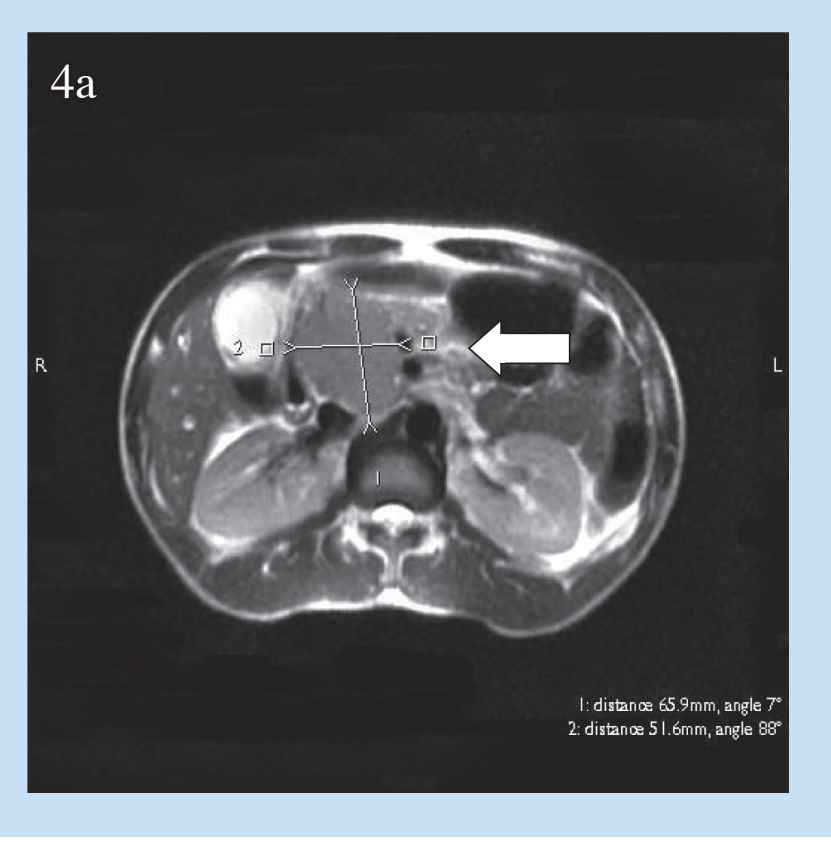

evaluation of indications for diagnostic and therapeutic ERCP is not yet finalised, the usefulness of ERCP stands beyond debate. As diagnostic ERCP is used less and less, the widespread use of the technique could be restricted to expert centres where skill in therapeutic ERCP and management of complications are available.

For diagnosis, MRCP offers sufficient advantages over ERCP to be considered as the initial modality. There is no risk of complications associated with the procedure of cannulation of the pancreatic duct and diagnostic yield is similar in most pathology. MRCP exceeds the possibilities of ERCP in providing additional information by cross-
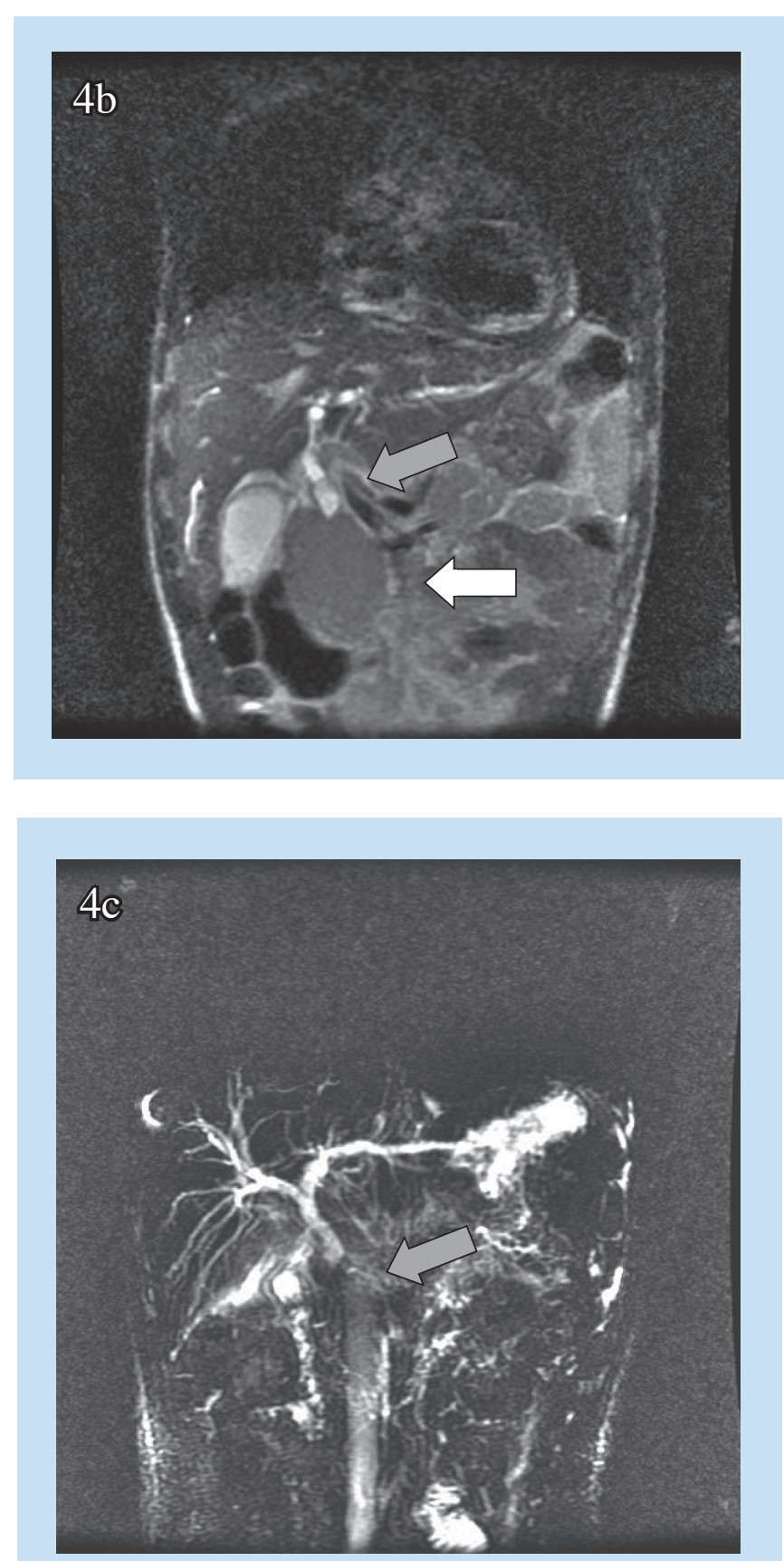

Figs $4 a, b$ and c. MRls demonstrating stenosis of the common bile duct (grey arrows) due to a tumour in the head of the pancreas (white arrow). Fig. 4a: Axial SSFSE T2,

Fig. 4b: Coronal SSFSE T2; Fig. 4c: High-resolution thick slab long TE $1100 \mathrm{~ms}$.

sectional MRI and MR angiography. There is no exposure to ionising radiation or to potentially risky iodinated contrast medium, and sedation is seldom indicated. Patients should be fasting and the procedure takes a few minutes, usually without sedation. The main potential problems with MRCP are image artifacts and difficulty in patient compliance because of claustrophobia. Image artifacts can be seen as bright signals arising from stationary fluid within the adjacent duodenum, duodenal diverticulae and ascitic fluid. In addition, local areas of signal dropout can be caused 


\section{ORIGINAL ARTICLE}
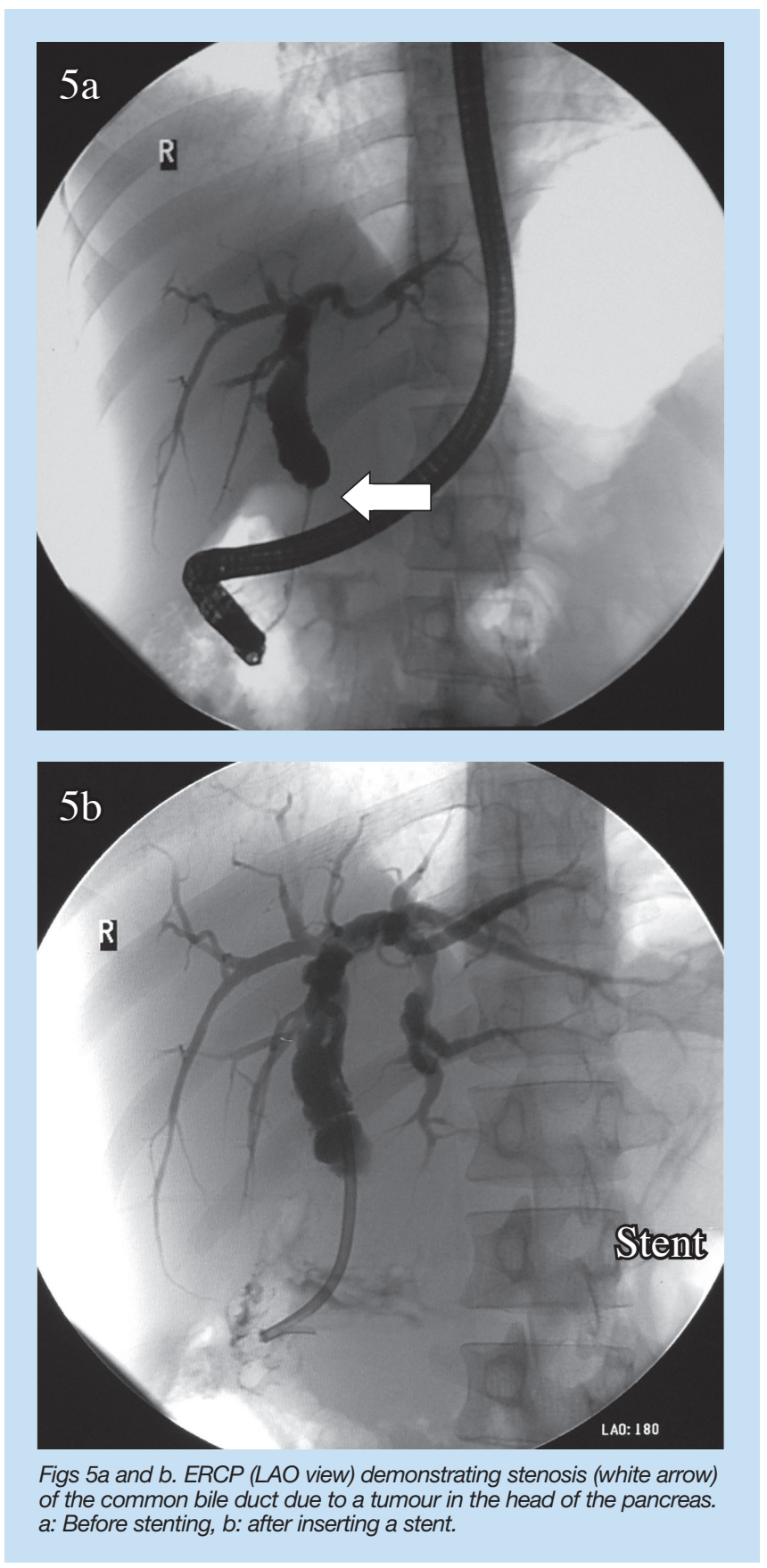

by metallic clips following cholecystectomy, defects induced by the right hepatic artery crossing the bile duct, or from severely narrowed ducts, such as occurs in primary sclerosing cholangitis (PSC).

Currently, MRCP has poorer resolution than direct cholangiography and can miss small stones $(<4 \mathrm{~mm})$, small ampullary lesions, primary sclerosing cholangitis, and strictures of the ducts. MRCP also has difficulty visualising small stones in the pancreatic duct. Obstructing stones are generally easier to identify than non-obstructing stones (especially if smaller than the thickness of the acquired image slices). Small stones may not be distinguishable from sludge, mucin or even blood. Stones $>4 \mathrm{~mm}$ are readily seen but cannot be differentiated from filling defects such as blood clots, tumour, sludge or parasites. Other mimickers of choledocholithiasis include flow artifacts, biliary air and a pseudocalculus at the ampulla. ${ }^{3}$

In the light of the current data, MRCP has the potential to replace diagnostic ERCP and thereby avoid possible complications related to ERCP. The accuracy of MRCP has been evaluated by several authors, with overall sensitivity of $85-97 \%$, specificity of $75-98 \%$, positive predictive values of $83-89 \%$, and negative predictive values of 82 $98 \%{ }^{4-6}$

A recent systemic review analysed the results according to underlying pathology. The median sensitivity for the 13 studies of choledocholithiasis was 0.93 (range $0.81-1.00)$ and the median specificity $0.94(0.83-0.99)$. For malignancy, sensitivity was $81-94.4 \%$ and specificity $92-100 \%$. The sensitivity to biliary dilatation was $87-100 \%$ and specificity 91 - $100 \%$. For obstruction, both sensitivity and specificity were 91 $100 \%$. Sensitivity for strictures was $100 \%$ and specificity $98-99 \%$. The review concluded that there is some evidence that MRCP is an accurate investigation compared with diagnostic ERCP, although the values for malignancy compared with choledocholithiasis were somewhat lower. ${ }^{3}$

Another systematic review that included a total of 67 studies found that the overall sensitivity and specificity of MRCP for the diagnosis of biliary obstruction were $95 \%$ and $97 \%$ respectively. Sensitivity was lower for stones (92\%) and for malignant conditions $(88 \%)^{7}{ }^{7}$

In a recent prospective study, 33 patients with jaundice due to bile duct strictures were examined by ERCP plus intraductal ultrasound (IDUS) v. MRCP. Diagnostic image quality for ERCP was $88 \%$ against $76 \%$ for MRCP $(p>0.05)$. Comparing ERCP and MRCP, complete depiction of the biliary tract was achieved in $94 \%$ and $82 \%$, respectively ( $p>0.05)$. ERCP and MRCP allowed correct differentiation of malignant from benign lesions in 76\% and 58\% ( $p=0.057$ ) of cases, respectively. By supplementing ERCP with IDUS, the accuracy of correct differentiation of malignant from benign lesions increased significantly to $88 \%$ ( $p=$ $0.0047)^{8}$

A study was undertaken by Park et al. to evaluate criteria retrospectively for differentiating extrahepatic bile duct cholangiocarcinoma from benign causes of stricture at MRCP, and to compare diagnostic accuracy of this modality with ERCP. The study concluded that accuracy of MRCP is comparable with that of ERCP. Regardless of modality, a lengthy segment of extrahepatic bile duct stricture with irregular margin and asymmetric narrowing suggests cholangiocarcinoma, while a short segment with regular margin and symmetric narrowing supports a benign cause. ${ }^{9}$

In another study (Moon et al.), 32 patients with suspected biliary pancreatitis were studied prospectively. MRCP was performed immediately before ERCP by separate blinded examiners within 24 hours of admission. The sensitivities of MRCP and ERCP for identifying choledocholithiasis were $80.0 \%$ and $90.0 \%$, respectively. The overall agreement between MRCP and ERCP was $90.6 \%$ for choledocholithiasis $(p<0.01)$. The sensitivity of MRCP in detecting choledocholithiasis decreased with dilated bile ducts (with a bile duct diameter $>10 \mathrm{~mm}$ it was $72.7 \%$, versus $88.9 \%$ in ducts $\leq 10 \mathrm{~mm}$ ). ${ }^{10}$

In our unit, MRI only supports single-shot fast spin-echo sequences. Newer variants of MRCP, including rapid acquisition with relaxation enhancement (RARE) and half-Fourier acquisition single-shot turbo 
spin-echo (HASTE), provide superior images. Single-shot RARE and HASTE techniques can be performed in a breath-hold period with a scan time of $<20$ seconds.

The optimal protocol to perform MRCP has not been defined and there continues to be variation across centres. As a general rule, the protocol depends upon the specific MR magnet being used, including its field strength (e.g. $1.5 \mathrm{v} .3 \mathrm{~T}$ ) and the manufacturer, as well as institutional experience and preferences. However, all acquisition protocols obtain heavily T2-weighted images as thick slabs and the images are reformatted in planes to optimise depiction of the hepatic and pancreatic ducts. Volume-rendered images may be used to depict the intra- and extrahepatic bile ducts.

In our study, MRCP had sensitivity, specificity, and positive and negative predictive values of $87 \%, 80 \%, 83.3 \%$ and $84.2 \%$, respectively, for bile duct calculi, which correlates well with results obtained in other parts of the world. It is known that the sensitivity of MRCP for detecting choledocholithiasis decreases with bile duct dilatation $(72.7 \%$ for bile duct diameters $>10 \mathrm{~mm} \mathrm{v.} 88.9 \%$ for diameters $<10 \mathrm{~mm}$ ). ${ }^{10}$ Of our 3 patients with false-negative results on MRCP, 2 had dilated bile ducts of $8 \mathrm{~mm}$ and $9 \mathrm{~mm}$, respectively.

For strictures, MRCP yielded sensitivity, specificity, and positive and negative predictive values of $33.3 \%, 96.6 \%, 80 \%$ and $77.8 \%$, respectively. Our sensitivity is substantially lower than international results. A systemic review reported a sensitivity of $100 \%$ for strictures. ${ }^{2}$ The review does not specifically mention the MRCP-protocol applied in each study. The reason for the low sensitivity in our study may be a result of the lower resolution of MRCP than direct cholangiography; it may also be the small number of our patients who definitely had strictures (12 out of 52 patients). If more advanced MRI sequences were used in the review, this could explain their high levels of sensitivity.

For bile duct occlusion, the sensitivity, specificity, and positive and negative predictive values were $50 \%, 78.9 \%, 11.1 \%$ and $96.8 \%$, respectively. Only 2 of our 52 patients had definite bile duct occlusion, explaining the low statistical power. Internationally, sensitivity and specificity ranges from $91 \%$ to $100 \%{ }^{2}$

The sensitivity, specificity, and positive and negative predictive values for bile duct dilatation were $52.9 \%, 77.8 \%, 90 \%$ and $30.4 \%$, respectively. Elsewhere, values for sensitivity and specificity are between $87 \%$ and $100 \%$, and $91 \%$ and $100 \%$, respectively. Of the 34 affected patients, 16 false-negative results were obtained for MRCP. The possible reason for the false-negative results may be that the reporting was performed by a general radiologist.

Overall, only 2 patients had proven pancreatic duct pathology on ERCP, again making the results difficult to interpret.

\section{Conclusion}

The aim of our study was to analyse the performance of MRCP as a diagnostic tool at our institution, using specificity, sensitivity, and positive and negative predictive values. We concluded that MRCP has high diagnostic accuracy solely for bile duct calculi. Owing to a small study population, results for other biliary pathology were inconclusive. Consequently, we propose MRCP as the method of choice for the diagnostic imaging of bile duct calculi. ERCP is reserved for therapeutic intervention in this setting.

It is well proven in current literature that MRCP has the potential to replace diagnostic ERCP in a wide range of bile duct abnormalities (tumour, stricture, occlusion), thereby avoiding possible complications related to ERCP. At our institution, further study with more patients is needed in future to reach the same conclusion. ERCP is still employed in the diagnosis of these abnormalities.

1. Bilbao MK, Dotter CT, Lee TG, et al. Complications of ERCP; a study of 10000 cases. Gastroenterology 1986; 70:314-320.

2. Kaltenthaler E, Bravo Vergel Y, Chilcott J, et al. A systematic review and economic evaluation of magnetic resonance cholangiopancreatography compared with diagnostic endoscopic retrograde cholangiopancreatography. Executive summary. Health Technology Assessment 2004; 8(10): 1-6.

3. David V, Reinhold C, Hochman M, et al. Pitfalls in the interpretation of MR cholangiopancreatography. AJR Am J Roentgenol 1998; 170: 1055-1059.

4. Taylor ACF, Little AF. Prospective assessment of MRCP for non-invasive imaging of the biliary tree. Gastrointest Endosc 2002; 55(1): 17-22.

5. Soto JA, Yucel EK. MRCP: Comparison with ERCP. Gastroenterology 1996; 110: 589-597.

6. Varghese JC, Farrell MA, Courtney G, et al. A prospective comparison of magnetic resonance cholangiopancreatography with endoscopic retrograde cholangiopancreatography in the evaluation of patients with suspected biliary tract disease. Clin Radiol 1999; 54: 513-520.

7. Romagnuolo J, Bardou M, Rahme E, et al. Magnetic resonance cholangiopancreatography: a meta-analysis of test performance in suspected biliary disease. Ann Intern Med 2003; 139: 547-557.

8. Domagk D, Wessling J, Reimer P, et al. Endoscopic retrograde cholangiopancreatography, intraductal ultrasonography, and magnetic resonance cholangiopancreatography in bile duct strictures: a prospective comparison of imaging diagnostics with histopathological correlation. Am J Gastroenterol 2004; 99(9): 1684-1689.

9. Mi-Suk Park, Tae Kyoung Kim, Kyoung Won Kim, et al. Differentiation of extrahepatic bile duct cholangiocarcinoma from benign stricture: Findings at MRCP versus ERCP. Radiology 2004; 233: 234240 .

10. Jong Ho Moon, Young Deok Cho, Sang Woo Cha, et al. The detection of bile duct stones in suspected biliary pancreatitis: Comparison of MRCP, ERCP, and intraductal US. Am J Gastroenterol 2005; 100: 1051-1057. 